\title{
An Optimization Method of Time Window Based on Travel Time and Reliability
}

\author{
Fengjie Fu, ${ }^{1}$ Dongfang Ma, ${ }^{1,2}$ Dianhai Wang,, and Wei Qian ${ }^{3}$ \\ ${ }^{1}$ College of Civil Engineering and Architecture, Zhejiang University, Hangzhou, Zhejiang 310058, China \\ ${ }^{2}$ Ocean College, Zhejiang University, Hangzhou, Zhejiang 310058, China \\ ${ }^{3}$ Hangzhou Hualong Traffic Survey and Design Ltd., Hangzhou, Zhejiang 310000, China
}

Correspondence should be addressed to Dianhai Wang; wangdianhai@zju.edu.cn

Received 15 July 2015; Revised 10 September 2015; Accepted 14 September 2015

Academic Editor: Jason Gu

Copyright (C) 2015 Fengjie Fu et al. This is an open access article distributed under the Creative Commons Attribution License, which permits unrestricted use, distribution, and reproduction in any medium, provided the original work is properly cited.

\begin{abstract}
The dynamic change of urban road travel time was analyzed using video image detector data, and it showed cyclic variation, so the signal cycle length at the upstream intersection was conducted as the basic unit of time window; there was some evidence of bimodality in the actual travel time distributions; therefore, the fitting parameters of the travel time bimodal distribution were estimated using the EM algorithm. Then the weighted average value of the two means was indicated as the travel time estimation value, and the Modified Buffer Time Index (MBIT) was expressed as travel time variability; based on the characteristics of travel time change and MBIT along with different time windows, the time window was optimized dynamically for minimum MBIT, requiring that the travel time change be lower than the threshold value and traffic incidents can be detected real time; finally, travel times on Shandong Road in Qingdao were estimated every 10 s, 120 s, optimal time windows, and $480 \mathrm{~s}$ and the comparisons demonstrated that travel time estimation in optimal time windows can exactly and steadily reflect the real-time traffic. It verifies the effectiveness of the optimization method.
\end{abstract}

\section{Introduction}

The time window determines the period of time that should be considered when estimating the current traffic information [1]. The selection of a time window meant that the traffic information, such as travel time, would be reestimated every time window. Most travel time estimation or prediction method adopted a constant time window subjectively: the time window was set as $2 \mathrm{~min}$ in the TransGuide algorithm [2]; TranStar algorithm estimated travel times every $30 \mathrm{~s}$; Transmit algorithm used a time window of $15 \mathrm{~min}$ [3]; Ma and Koutsopoulos demonstrated that a larger time window gave smoother travel time with longer delay and a smaller one reduced delay with volatile travel time [4]. Sun and Zhang divided the travel time prediction model into two components based on the corresponding signal state: a green phase model with three different time windows and a red phase model with the time window equal to red time. Nevertheless, the values of the four time windows merely depended on the cycle length and were not the optimal [5].
Jiang et al. presented an optimization method of the time window of floating car data for the minimum relative errors and delayed rates of ILTT (Individual Link Travel Time) [6]. However, the method determined the optimal value simply based on the relationship between the quality of the traffic information and the time window. Therefore, it is necessary to propose optimizing indexes and an optimization algorithm to get the optimal time window for travel time estimation and prediction.

Jackson and Jucker found that there was a trade-off between the result of the travel time estimation or prediction and travel time reliability [7]. Many studies defined travel time reliability on the basis of the mean, standard deviation, and percentiles [8-10]. For example, the Federal Highway Administration (FHWA) presented the five measures of travel time reliability: (1) the 90th or the 95th percentile travel time; (2) the travel time index (TTI), defined as the ratio of the mean to the free-flow travel time; (3) the Buffer Time Index (BTI), namely, the difference between the 95th percentile and the mean divided by the mean; it indicates 
the amount of extra time needed to be on time for $95 \%$ of trips; (4) the planning time index (PTI), namely, the ratio of the 95th percentile travel time to the free-flow travel time; (5) congestion frequency, defined as the ratio of congestion period to the total days [11]. But Van Lint and Van Zuylen indicated that indexes based on the mean and standard deviation may not reflect travel time reliability well since the travel time distribution showed significant skewness. So they proposed the so-called skew-width method composed of the skewness of the travel time and the width of travel time [12]. And Emam and Al-Deek pointed out that log-normal distribution emerged as a useful model representing travel time by examining the use of different statistical distributions including log-normal, exponential, gamma, and Weibull distributions for empirical travel time data [13]. However, many research found that there was some evidence of bimodality in the actual travel time distributions and developed many models for estimating the bimodal distribution [14-16]. Yang et al. demonstrated that a constant bimodal distribution could not always provide good fit for travel times of interrupted flow on different urban roads or during different periods because of the traffic state on the road, the delay at the downstream intersection, peak hours, and so on. They modeled six types of bimodal distributions which applied normal and lognormal distribution as typical distribution to fit RFID (Ratio Frequency Identification) data in Nanjing. The distribution could distinguish the travel time samples of the rapid flow and slow flow and provided the foundation for redefinition of travel time reliability [17]. However there exists inconsistency in the mixture model of normal and lognormal distribution because of the different units. Then the use of different dimensions in the data in the model can lead to different results [18].

The objective of this research is to develop a method to optimize the value of time window on the basis of travel time and reliability. The remainder of the paper is organized as follows: the second section outlines the traffic data source and travel time calculation; the third section proposes a new index reflecting travel time reliability in accord with the bimodal distribution; in the fourth section, an optimization method is presented to obtain the optimal time window; finally, the fifth section gives numerical experiments to demonstrate effectiveness of the optimization method and the optimal time window.

\section{Study Data}

2.1. Data Collection System. The Multifunction Automatic Detecting and Recording System is installed at intersections with video pattern and capture pattern, so it can not only detect and capture illegal driving but also monitor and record all passing vehicles. The front-end devices transfer videos and images to the back-end computer; and then traffic data, including plate number, intersection ID, departure time, departure lane ID, departure entrance ID, departure speed, and position obtained by using ANNR, is available. The collected traffic information are stored as database, pictures, and videos. The data collection system becomes wide and the installation is as shown in Figure 1.

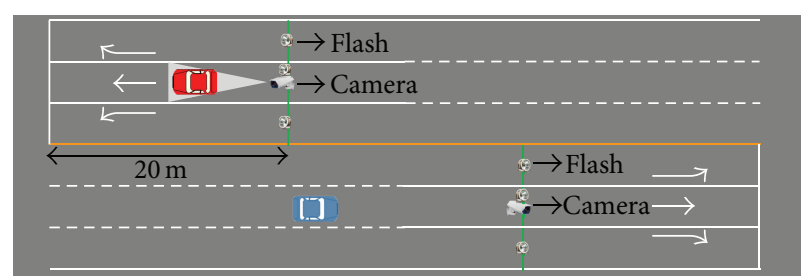

FIgURE 1: Installations of the Multifunction Automatic Detecting and Recording System.

2.2. Data Precision. Travel time information data used in the paper is collected from cameras at intersections on the Shandong Road in Qingdao, from December 1, 2014, to December 31, 2014. Firstly, traffic data was collected at Shandong-Jiangxi Intersection, including database and videos, for analyzing the practical detection precision of the system. Figure 2(a) shows time-of-day fluctuations of the number of identified vehicles (IV), passing traffic volumes $(\mathrm{PV})$, and their average percentage during 7:00-19:00 on December 22, 2014, at the southern approach. The number of identified vehicles (i.e., vehicles captured and recorded and license plate positioned, including ones with license plate numbers incorrectly recognized) is calculated from the database and passing traffic volume is observed from videos by manual counting. A typical time-of-day change with two peaks can be found in the passing traffic volumes and the number of identified vehicles. The great fluctuations between time windows are due to the signal control. The average percentage of IV with PV without considering the errors of the identified plate number is about $93.28 \%$. The accuracy of plate number can be larger than $90 \%$ once identified and the software makers have verified it. Moreover, it went dark after 5:30 and the percentage is still high with the flash or everlasting lamp working as shown in Figure 2(b). Therefore, the author can demonstrate that the real average sample rate can be up to $85.8 \%$, and it can be guaranteed at night with the flash or everlasting lamp working.

2.3. Travel Time Acquisition. The travel time of a vehicle on a link, namely, the time difference from the upstream $(A)$ to downstream $(B)$ intersection, is computed as shown in (1) by matching the plate number from a pair of cameras $A$ and $B$ :

$$
T_{i}=t_{i}^{B}-t_{i}^{A}
$$

where $t_{i}^{A}$ and $t_{i}^{B}$ are the time that vehicle $i$ passes the stop line at the upstream and downstream intersection, respectively and $T_{i}$ is the travel time of vehicle $i$ on the link.

\section{Travel Time Reliability}

3.1. The Basic Unit of Time Window. Points in Figures 3(a) and 3 (b) indicate travel times and departure times of all vehicles during the four different periods (22:00-23:00, 7:30-8:30, 13:00-14:00, and 17:30-18:30) of day on the Shandong Road from Shandong-Jiangxi Intersection to Shandong-Minjiang Intersection and from Shandong-Minjiang Intersection to 


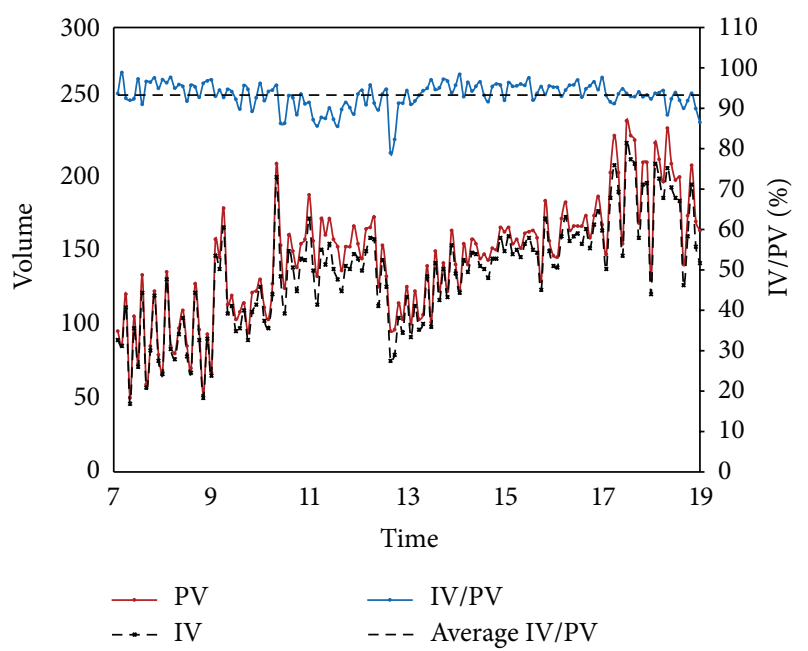

(a)

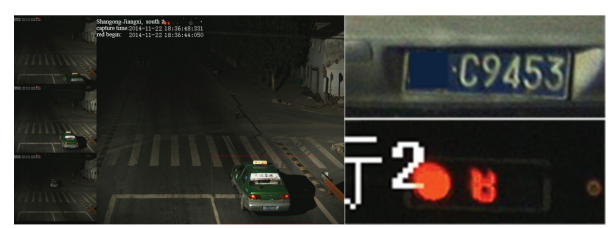

(b)

FIgURE 2: (a) The detection precision result at Shandong-Jiangxi Intersection during 7:00-19:00 on December 22, 2014 and (b) pictures captured by the system at 18:36.

Shandong-Jiangxi Intersection separately. It was obvious that traffic flow was interrupted and travel times showed cyclic change due to traffic state, signal timing, and signal cycle length. Therefore, the signal cycle length at the upstream intersection should be set as the basic unit of the time window for travel time estimation and prediction.

3.2. Travel Time Distribution. It can be seen that points surrounded by a red outline in Figure 3 were composed of one cluster or multiclusters: (1) vehicles can pass through the downstream intersection without signal delay or wait for at most a short duration of the red phase during 22:00-23:00 and 13:00-14:00 because of unsaturated traffic condition and short cycles. Therefore, travel time data fell into no more than two clusters and each cluster was centered. (2) Some vehicles may wait for two or more red lights during 7:308:30 and 17:30-18:30 because of the great traffic demand and oversaturated traffic state. Consequently, travel time points can be divided into two or more groups and each group was discrete. A bimodal distribution is fitted against the travel time data in each cycle. The model of the bimodal distribution is as presented in

$$
\begin{aligned}
f(T T)= & \omega_{1} \frac{1}{\sqrt{2 \pi} \sigma_{1}} \exp \left(\frac{\left(T T-\mu_{1}\right)^{2}}{2 \sigma_{1}^{2}}\right) \\
& +\omega_{2} \frac{1}{\sqrt{2 \pi} \sigma_{2}} \exp \left(\frac{\left(T T-\mu_{2}\right)^{2}}{2 \sigma_{2}^{2}}\right),
\end{aligned}
$$

where $f(T T)$ is the probability density function of travel time; TT indicates the estimation value of travel time; $\mu$ expresses the mean of the travel time distribution and $\mu_{1}$ is the larger one; $\sigma^{2}$ is the variance of travel time with $\sigma_{1}$ and $\sigma_{2}$ corresponding to $\mu_{1}$ and $\mu_{2}$, respectively; likewise, $\omega_{1}$ and $\omega_{2}$, namely, the ratio of each subsample to the whole sample, correspond to $\mu_{1}$ and $\mu_{2}$ separately with the sum of them equal to 1 .

Parameters in (2) are estimated using the Expectation Maximization (EM) algorithm, and the process is as follows:

(1) Initialize the parameters $\theta(\mu, \sigma, \omega)$ of the Gaussian mixture distribution model.

(2) E-step: given the current estimate of the parameters in step (1), the conditional distributions of the two distributions $p(i, 1)$ and $p(i, 2)$ are determined by Bayes theorem to using their own probability density functions $g\left(T T_{i} \mid \mu_{1}, \sigma_{1}\right)$ and $g\left(T T_{i} \mid \mu_{2}, \sigma_{2}\right)$ weighted by $\omega_{1}$ and $\omega_{2}($ see $(3))$.

(3) The M-step computes parameters maximizing the expected log-likelihood found on the E-step. The estimation results of the first distribution with $m$ samples are presented as shown in (4):

$$
\begin{aligned}
p(i, 1) & =\frac{\omega_{1} g\left(T T_{i} \mid \mu_{1}, \sigma_{1}\right)}{\omega_{1} g\left(T T_{i} \mid \mu_{1}, \sigma_{1}\right)+\omega_{2} g\left(T T_{i} \mid \mu_{2}, \sigma_{2}\right)}, \\
p(i, 2) & =\frac{\omega_{2} g\left(T T_{i} \mid \mu_{2}, \sigma_{2}\right)}{\omega_{1} g\left(T T_{i} \mid \mu_{1}, \sigma_{1}\right)+\omega_{2} g\left(T T_{i} \mid \mu_{2}, \sigma_{2}\right)} \\
\mu_{1} & =\frac{1}{\omega_{1}} \sum_{i=1}^{m} p(i, 1) T T_{i}, \\
\sigma_{1}^{2} & =\frac{1}{\omega_{1}} \sum_{i=1}^{m} p(i, 1)\left(T T_{i}-\mu_{1}\right)\left(T T_{i}-\mu_{1}\right)^{T} \\
\omega_{1} & =\sum_{i=1}^{m} p(i, 1) .
\end{aligned}
$$

(4) Repeat from step (1) again until the value of $\| \theta^{N}-$ $\theta^{N-1} \|$ meets the threshold.

Consequently, the bimodal distributions of travel times in the certain signal cycles surrounded by a red outline in Figure 3 were got using the EM algorithm. Figure 4 gives their frequency histogram and probability density curve. The results are tested by K-S (Kolmogorov-Smirnov) test. The null hypothesis is that the samples have a standard normal distribution. The alternative hypothesis is that the samples do not have that distribution. The test accepts the null hypothesis if the significance probability Prob is larger than the significance level and rejects it otherwise. As Table 1 shows, the values of Prob in the K-S test were all larger than 0.05. It indicates that the samples do have a standard normal distribution at 0.05 significance level and the bimodal distribution results fit well. 
TABLE 1: The results of K-S test.

\begin{tabular}{lcccccccc}
\hline Data & Figure 4(a)-1 & Figure 4(a)-2 & Figure 4(a)-3 & Figure 4(a)-4 & Figure 4(b)-1 & Figure 4(b)-2 & Figure 4(b)-3 & Figure 4(b)-4 \\
\hline$N$ & 28 & 154 & 77 & 48 & 58 & 100 & 74 & 225 \\
Prob & 0.11 & 0.39 & 0.17 & 0.21 & 0.23 & 0.18 & 0.30 & 0.12 \\
\hline
\end{tabular}
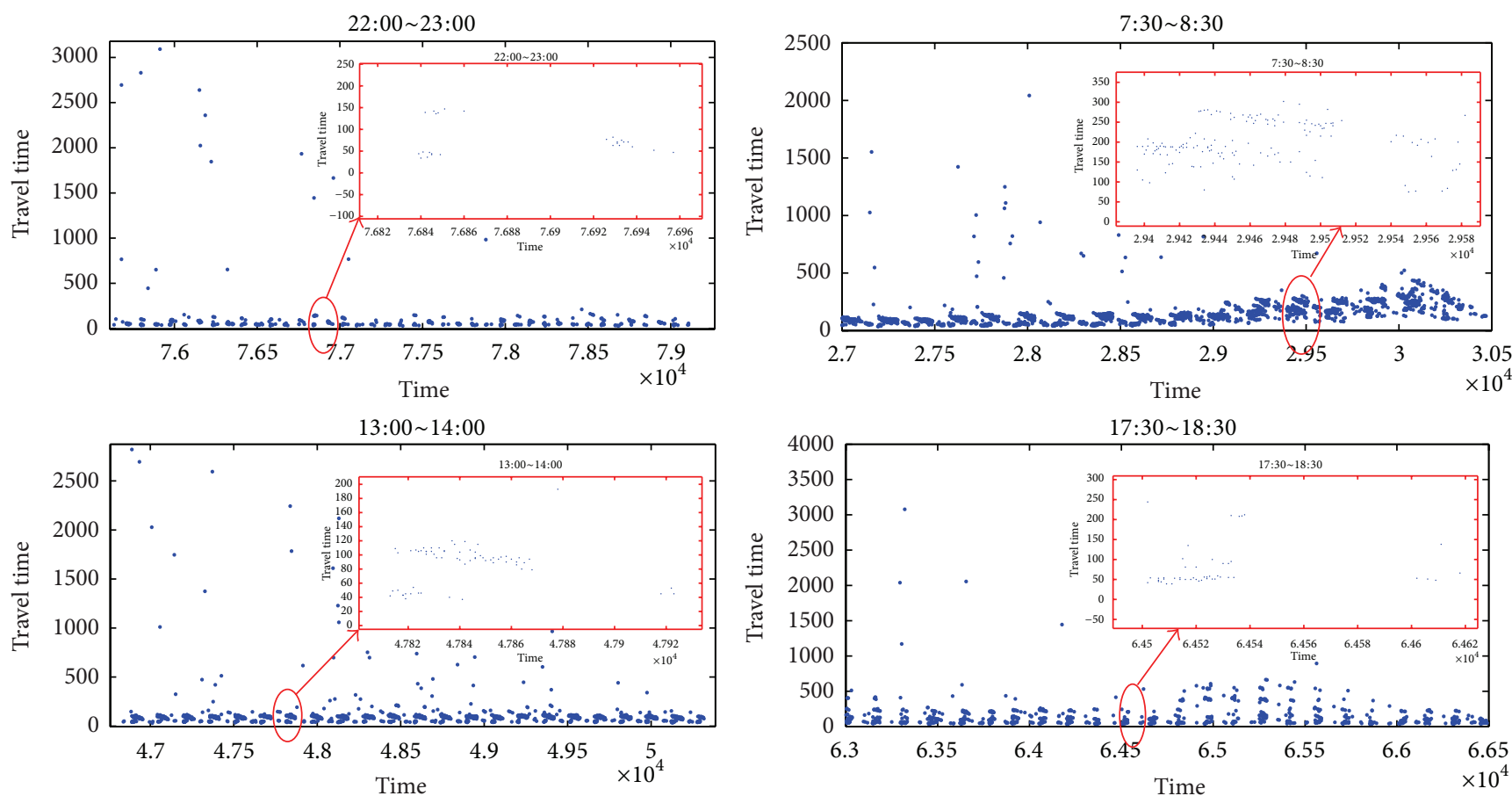

(a)
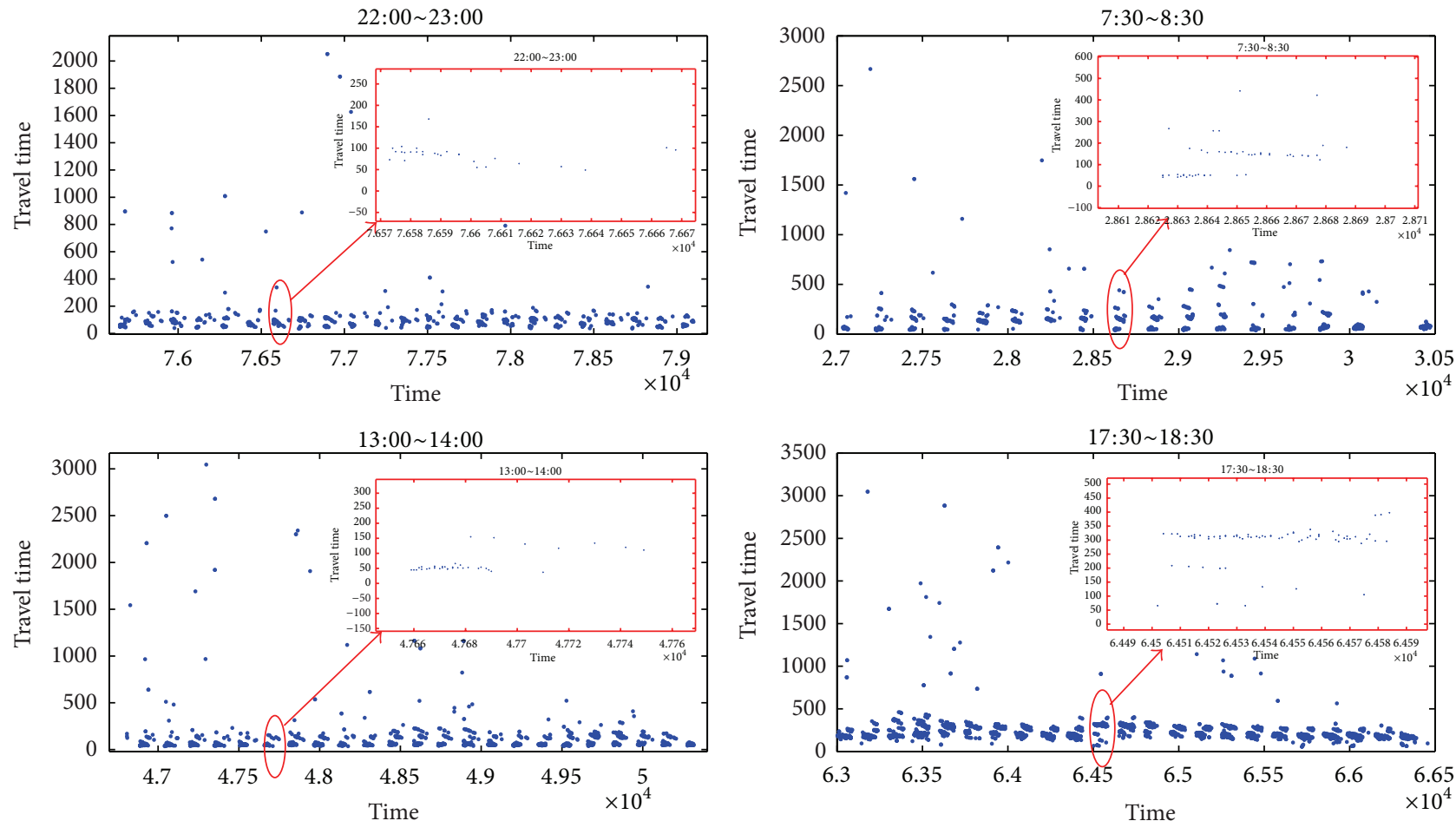

(b)

FIgURE 3: (a) Individual travel time along with time from Shandong-Jiangxi Intersection to Shandong-Minjiang Intersection and (b) individual travel time along with time from Shandong-Minjiang Intersection to Shandong-Jiangxi Intersection. 

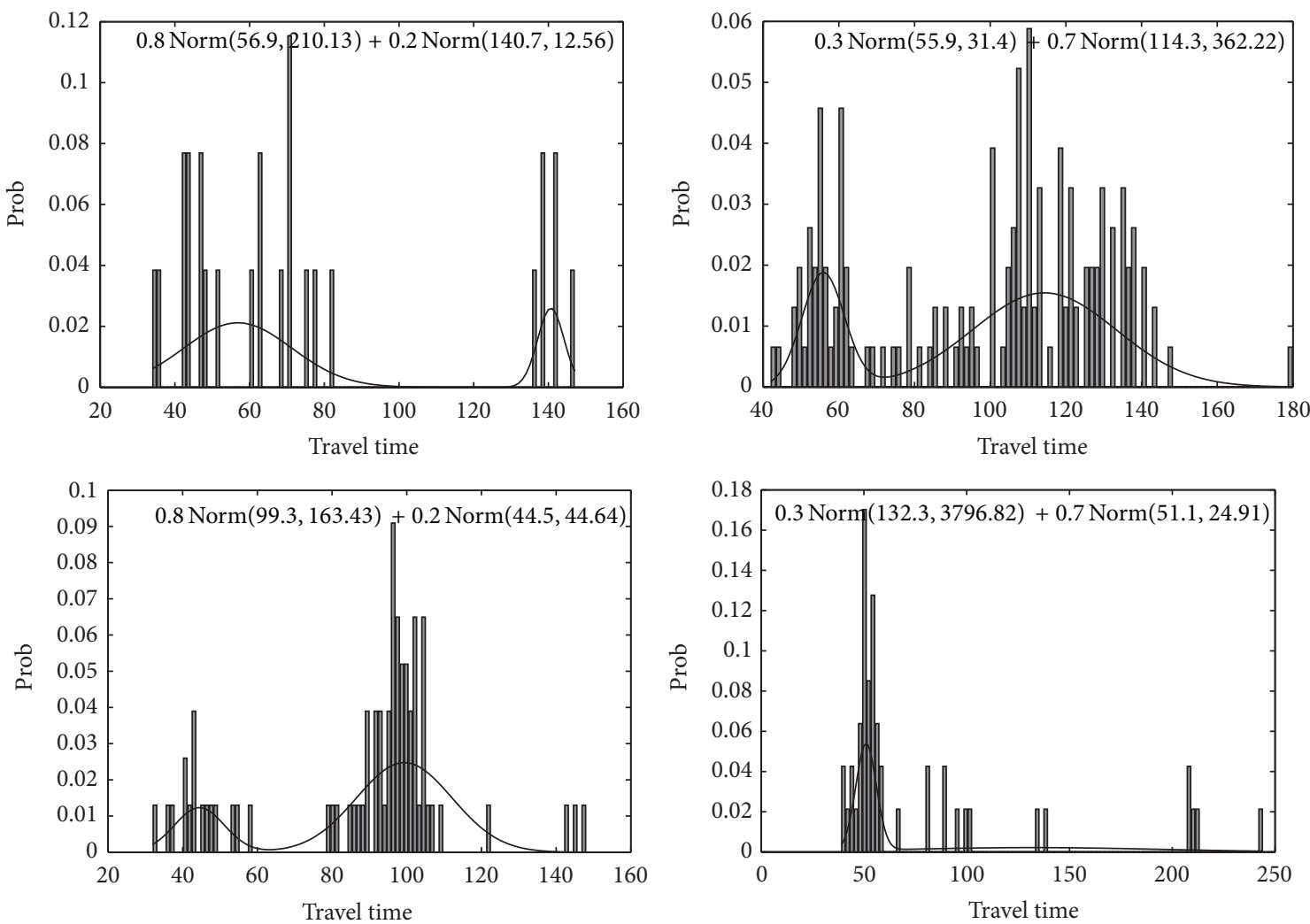

(a)
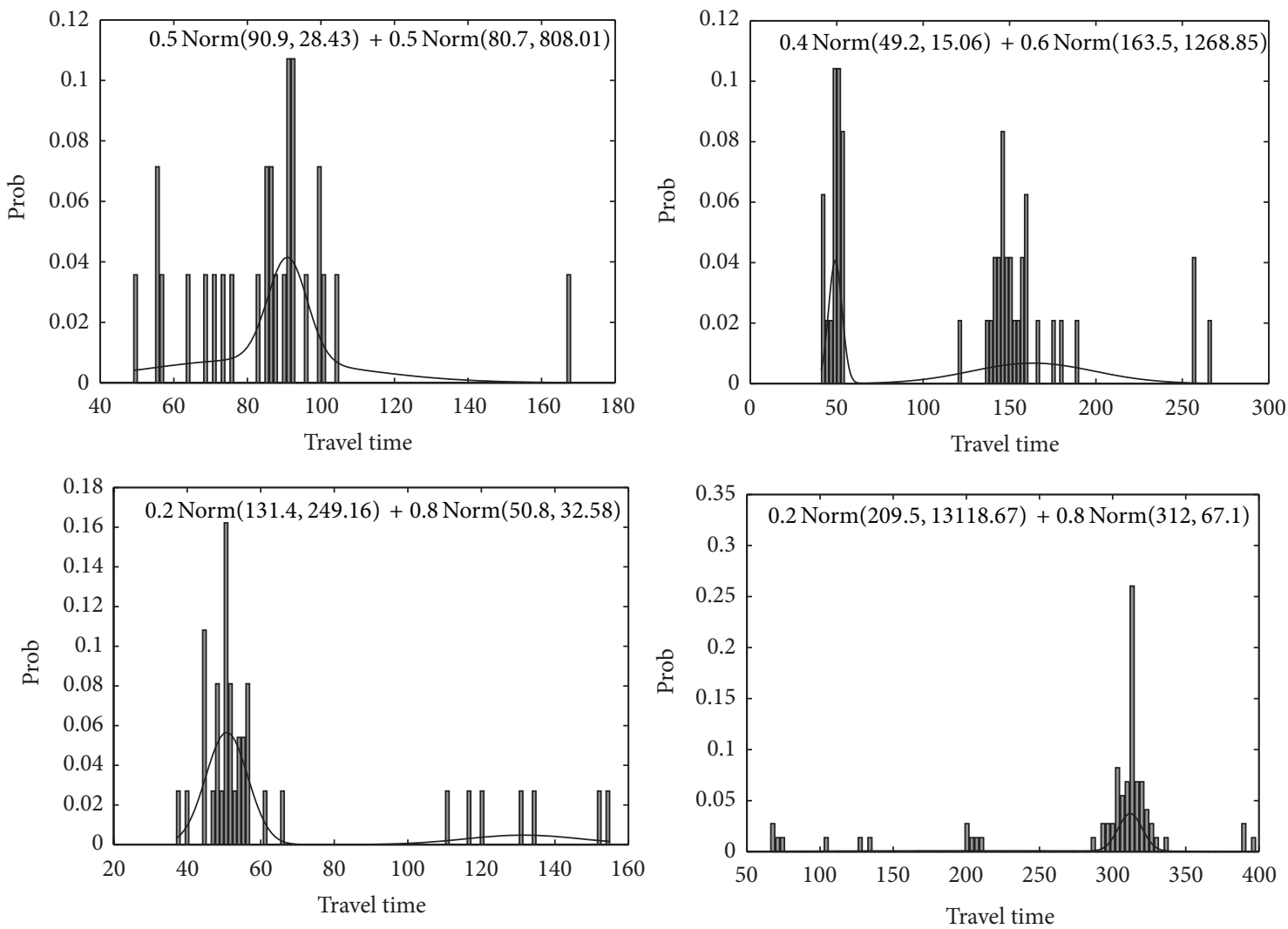

(b)

Figure 4: (a) Fitting results in a single cycle marked in Figure 3(a) (from Shandong-Jiangxi Intersection to Shandong-Minjiang Intersection) and (b) fitting results in a single cycle marked in Figure 3(b) (from Shandong-Minjiang Intersection to Shandong-Jiangxi Intersection). 


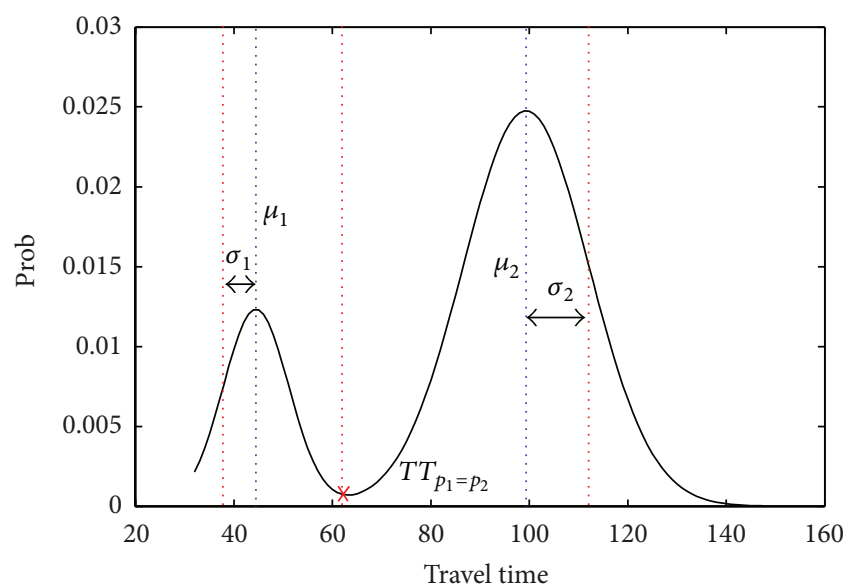

(a)

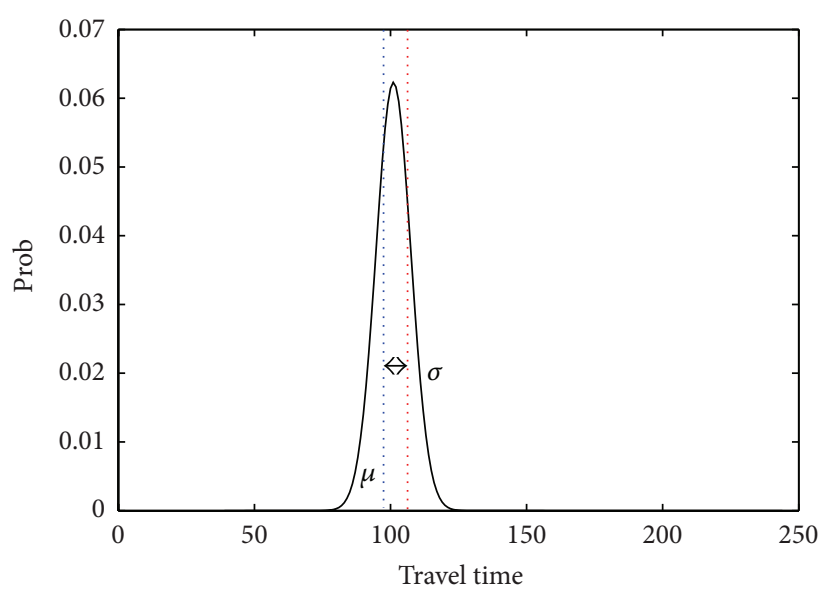

(b)

Figure 5: Parameters of travel time distribution.

3.3. Travel Time Reliability Index. A new travel time reliability index, the Modified Buffer Time Index, is proposed on the basis of the characteristic of the bimodal distribution and its common ground with the unimodal distribution as follows:

(1) When travel times are bimodal-distributed, the travel time estimation and MBIT are computed as presented in

$$
\begin{aligned}
T T & =\omega_{1} \mu_{1}+\omega_{2} \mu_{2}, \\
\operatorname{MBIT} & =\frac{\mu_{2}+\sigma_{2}-\left(\mu_{1}-\sigma_{1}\right)}{T T_{p_{1}=p_{2}}},
\end{aligned}
$$

where MBIT is the value of the Modified Buffer Time Index and $T T_{p_{1}=p_{2}}$ indicates the travel time estimation at the crossing point of the two distributions, namely, the estimation value when $p_{1}=p_{2}$ (see Figure 5).

(2) When travel times are unimodal-distributed, the computations of travel time estimation and MBIT are as follows:

$$
\begin{aligned}
\text { TT } & =\mu, \\
\text { MBIT } & =\frac{2 \sigma}{\mu} .
\end{aligned}
$$

\section{The Optimization of the Time Window}

4.1. The Optimization Index. As (7) presents, the value of $k$ th time window, $\mathrm{TW}_{k}$, is the sum of several cycles:

$$
\mathrm{TW}_{k}=\sum_{j=1}^{m_{k}} c_{j}
$$

where $m_{k}$ expresses the number of signal cycles in $k$ th time window and $c_{j}$ expresses the length of $j$ th signal cycle with $j$ smaller than $m_{k}$; when the signal timing plan is fixed and the cycle length is $c, \mathrm{TW}_{k}$ is equal to the product of $m_{k}$ and $c$.

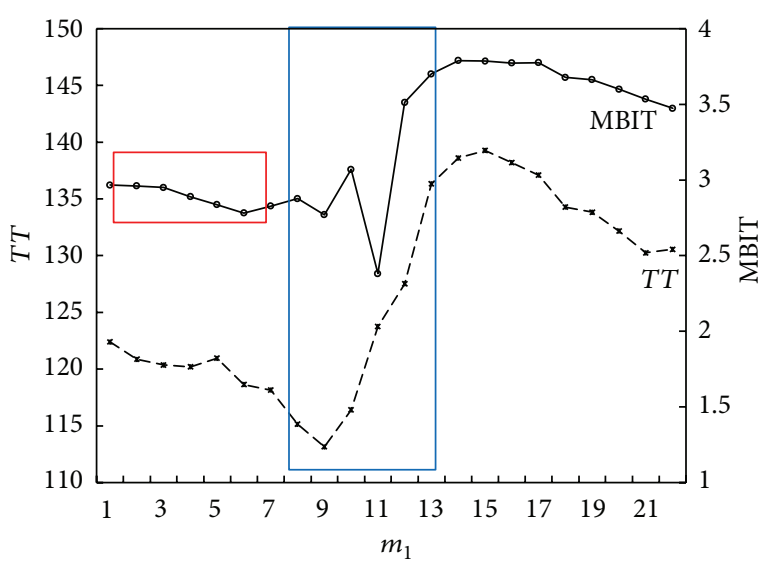

FIgURE 6: The variation of MBIT and TT with $m_{1}$.

Travel time information data during 17:30-18:30 on the link from Shandong-Jiangxi Intersection to ShandongMinjiang Intersection were analyzed to determine the optimization index. As Figure 6 shows, the values of MBIT surrounded by the red line reduced with the number of signal cycles in the first time window $\left(m_{1}\right)$, and then traffic demand changed greatly resulting in a great change in MBIT and TT (surrounded by the blue line).

Overall, when traffic demand changes greatly, traffic flow runs unstable and travel times change greatly, so a larger time window reduces the travel time reliability instead and cannot reflect the real change of the traffic condition. Although traffic flow runs stable with stable travel times when the traffic demand change little, a larger time window cannot ensure timely response to traffic events. However, the two conditions have one thing in common: the travel time reliability in a small time window can be too low. Therefore, the optimization of the time window should be based on the travel time and its reliability. And it is realized by using 
the relative variation ratios of the travel time estimation and Modified Buffer Time Index $R_{T T}^{m_{k}}$ and $R_{\mathrm{MBIT}}^{m_{k}}$ as presented in

$$
\begin{aligned}
R_{T T}^{m_{k}} & =\left|\frac{T T_{m_{k}+1}-T T_{k 1}}{T T_{k 1}}\right| \cdot 100 \%, \\
R_{\mathrm{MBIT}}^{m_{k}} & =\frac{\operatorname{MBIT}_{m_{k}+1}-\mathrm{MBIT}_{k 1}}{\operatorname{MBIT}_{k 1}} \cdot 100 \%,
\end{aligned}
$$

where $T T_{k 1}, T T_{m_{k}+1}$ are travel time estimations of the first signal cycle and $\left(m_{k}+1\right)$ th signal cycle in $k$ th time window separately and $\mathrm{MBIT}_{k 1}$ and $\mathrm{MBIT}_{m_{k}+1}$, respectively, indicate the Modified Buffer Time Index corresponding to $T T_{k 1}$ and $T T_{m_{k}+1}$.

Traffic events can be detected using the relative variation ratios of the travel time estimations in two successive time windows at least. So as (9) demonstrates, a timely response to traffic events requires the sum of the two successive time windows to be less than the minimum response time to traffic events:

$$
\Delta t_{k}= \begin{cases}2 \sum_{j=1}^{m_{1}} c_{j}-T & k=1 \\ \mathrm{TW}_{k-1}+\sum_{j=1}^{m_{k}} c_{j}-T & k>1,\end{cases}
$$

where $\Delta t_{k}$ is the time difference between the sum of the two successive time windows and the minimum response time to traffic events; $T$ is the minimum response time to traffic events, and under urban roads it is taken between $15 \mathrm{~min}$ and $30 \mathrm{~min}$ [19]; $\mathrm{TW}_{k-1}$ indicates the value of $(k-1)$ th time window.

4.2. The Optimization Process. The values of $R_{T T}^{m_{1}}$ and $R_{\mathrm{MBIT}}^{m_{1}}$ when the number of signal cycles of the first time window $\left(m_{1}\right)$ ranges from 1 to 22 are as shown in Figure 7. On the one hand, $R_{\mathrm{MBIT}}^{m_{1}}$ was less than 0 when $m_{1} \leq 9$ and kept reducing when $m_{1} \leq 6$, so $m_{1}$ should not be larger than 6 to ensure a higher travel time reliability; on the other hand, $R_{T T}^{m_{1}}$ did not exceed $5 \%$ (for instance) until $m_{1}>7$ and $\Delta t_{1}$ did not exceed 0 until $m_{1}>3$, so $m_{1}$ should not be larger than 3 . In general, the optimization process on the basis of the variation of $R_{T T}^{m_{k}}$ and $R_{\mathrm{MBIT}}^{m_{k}}$ and the value of $\Delta t_{k}$ is as follows:

(1) The internalization: $k=1, m_{1}=1, R_{T T}^{m_{1}}=0, R_{\mathrm{MBIT}}^{m_{1}}=$ 0 .

(2) Calculate the values of $R_{T T}^{m_{k}}, R_{\mathrm{MBIT}}^{m_{k}}$, and $\Delta t_{k}$.

(3) The threshold judgment:

$$
\begin{aligned}
& \text { if } R_{\mathrm{TT}}^{m_{k}} \leq \varepsilon \& R_{\mathrm{MBIT}}^{m_{k}} \leq 0 \&\left(R_{\mathrm{MBIT}}^{m_{k}}-R_{\mathrm{MBIT}}^{m_{k-1}}\right) \leq 0, \\
& m_{k}=m_{k}+1, \text { and repeat from Step }(2) ; \\
& \text { if } R_{\mathrm{TT}}^{m_{k}}>\varepsilon\left|R_{\mathrm{MBIT}}^{m_{k}}>0\right| \Delta t_{k}>0 \mid\left(R_{\mathrm{MBIT}}^{m_{k}}-\right. \\
& \left.R_{\mathrm{MBIT}}^{m_{k-1}}\right)>0, m_{k}=m_{k}-1, k=k+1 \text {, and repeat } \\
& \text { from Step (1). }
\end{aligned}
$$

The value of $\varepsilon$ can be set according to traffic control requirements. It is taken as $5 \%$ in the paper and $T=15 \mathrm{~min}$. Then the optimal number of signal cycles in the first time

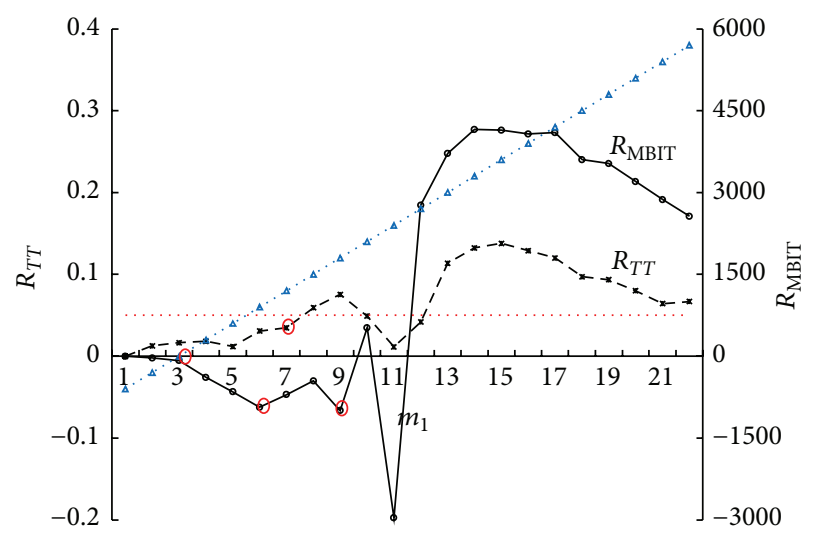

FIgURE 7: The variation of $R_{\mathrm{TT}}^{m_{1}}, R_{\mathrm{MBIT}}^{m_{1}}$, and $\Delta t_{1}$ with $m_{1}$.

window during 17:30-18:30 on the link from ShandongJiangxi Intersection to Shandong-Minjiang Intersection is equal to 3 , so the optimal time window is $450 \mathrm{~s}$.

\section{Empirical Analysis}

The optimal time windows during 17:30-18:30 on the link from Shandong-Jiangxi Intersection to Shandong-Minjiang Intersection can be obtained using the optimization method in Section 3 and the results are as presented in Table 2. Each column in the table gives the travel time reliability index values in each time window and the time window value. For example, in the second column, $k=2$ indicates that it is the optimization of the second time window and then $m_{k}$ should just be $m_{2} ; m_{2}$ increases gradually and when it is equal to 2 , $\Delta t_{k}, R_{T T}^{m_{k}}$, and $R_{\mathrm{MBIT}}^{m_{k-1}}$ do not exceed their thresholds, while $R_{\mathrm{MBIT}}^{m_{k}}$ is over $10 \%$, therefore $m_{2}$ should be set as 2, namely, $450 \mathrm{~s}$. Thus all the optimized time windows values can be got and they are shown as in the last two rows.

Figure 8 shows travel time estimations with the time window equal to $10 \mathrm{~s}$ and $120 \mathrm{~s}$, the optimal values of $\mathrm{TW}_{k}$, and $480 \mathrm{~s}$ from top to bottom. Red points denote travel time sample data of all vehicles on the road and each black bar shows the travel time estimation value in each time window. Time windows in all the cases except for the third one were set as fixed values. It can be seen from the figure that the widths of black bars in each of these three cases are the same except the one using the optimal values of $\mathrm{TW}_{k}$. When the time window was set as $10 \mathrm{~s}$ or $120 \mathrm{~s}$, travel time sample data in a single time window was so few that the travel time estimation was directly taken as the average travel time. Figure 8(a) presents that there existed gaps and great fluctuations among travel time estimations $(\mathrm{TW}=10 \mathrm{~s}$ ) since traffic flow was interrupted due to traffic signal control and the time window was too small. The difference of the sample data among each time window was big and correspondingly several travel time estimations ( $\mathrm{TW}=120 \mathrm{~s}$ ) were too large or too small and cannot demonstrate the real value, since TW was not a multiple of the signal cycle length and the travel times changed periodically with the signal timing, while a time window of $480 \mathrm{~s}$ was so large that there was hardly any 
TABLE 2: The optimal time intervals on Shandong Road during 17:30-18:30.

\begin{tabular}{lcccccccccccc}
\hline$k$ & 1 & 2 & 3 & 4 & 5 & 6 & 7 & 8 & 9 & 10 & 11 & 12 \\
\hline$\Delta t_{k}$ & $\mathbf{0}$ & -300 & -300 & -300 & -600 & -600 & 0 & $\mathbf{0}$ & -600 & -600 & -300 & -600 \\
$R_{T T}^{m_{k}}(\%)$ & 1.6 & 0.2 & $\mathbf{8 . 8}$ & $\mathbf{7 . 7}$ & $\mathbf{7 . 1}$ & 2.7 & $\mathbf{8 . 1}$ & 0.1 & 0.2 & $\mathbf{1 5 . 0}$ & $\mathbf{1 3}$ & 4.5 \\
$R_{\mathrm{MBIT}}^{m_{k-1}}(\%)$ & -0.2 & -3.3 & -12.4 & -22.1 & 0 & 0 & -5.6 & -34 & 0 & 0 & -3.6 & 0 \\
$R_{\mathrm{MBIT}}^{m_{k}}(\%)$ & -0.5 & $\mathbf{1 0 . 4}$ & -17.0 & -22.3 & -21 & $\mathbf{1 . 4}$ & -5.8 & -38 & $\mathbf{6 . 6}$ & -18.2 & -10.1 & $\mathbf{9 . 9}$ \\
$m_{k}$ & 3 & 2 & 2 & 2 & 1 & 1 & 3 & 3 & 1 & 1 & 2 & 1 \\
$\mathrm{TW}_{k}$ & 450 & 300 & 300 & 300 & 150 & 150 & 450 & 450 & 150 & 150 & 300 & 150 \\
\hline
\end{tabular}

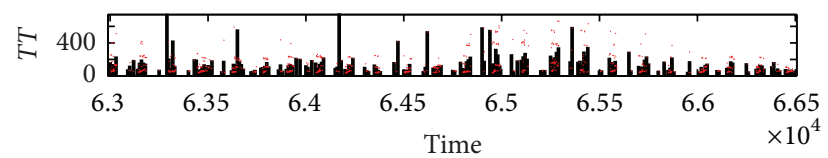

(a) $\mathrm{TW}=10 \mathrm{~s}$

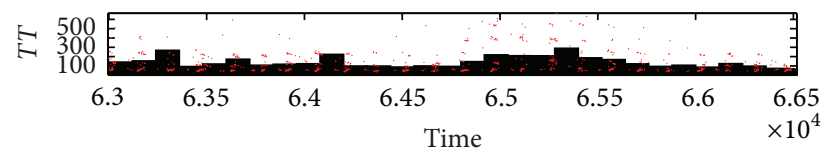

(b) $\mathrm{TW}=120 \mathrm{~s}$

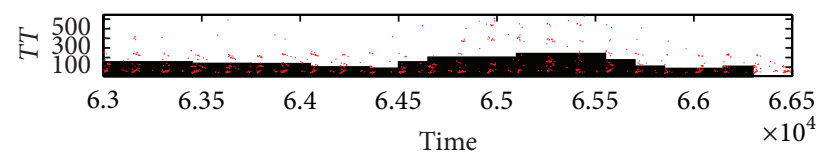

(c) TW $=$ The optimal values

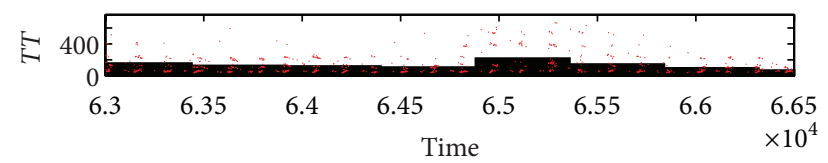

(d) $\mathrm{TW}=480 \mathrm{~s}$

FIGURE 8: Travel time estimations in different time intervals.

difference or change among travel time estimations and the result cannot make any sense to traffic state identification or traffic events detection. However, when the value of each time window was taken as the optimal one, the estimation result can reflect the change of the traffic condition with stable values as shown in Figure 8(c).

\section{Conclusion}

The authors provided an optimization model for the determination of the time window: firstly, by exploring the characteristic of the travel time bimodal distribution, the model used the means, standard deviations, and crossing point value of the two distributions to compute the Modified Buffer Time Index indicating the travel time reliability on urban roads; secondly, the model took the signal cycle length as the basic unit of the time window, the relative variation ratios of the travel time estimation, and the Modified Buffer Time Index as the optimizing index for a minimum travel time reliability and timely response to traffic events; finally, the empirical analysis verified the optimization method since travel time estimations using the optimal time window can reflect the variation of the real traffic state better with much stable values.

However, much more travel time information data should be collected and analyzed to confirm whether all the travel time distributions can be modeled by the six types of bimodal distributions applying normal and lognormal distribution. Correspondingly, the estimation of travel time and the computation of travel time reliability should be completed. Moreover, study on the relationship between the decision threshold and different urban roads should be added to realize the automatic determination of the decision threshold.

\section{Notations}

Variables:

$T_{i}: \quad \quad \quad$ The travel time of vehicle $i$ on the link

$t_{i}^{A}: \quad$ The time that vehicle $i$ passes the stop

$t_{i}^{B}$ line at the upstream intersection The time that vehicle $i$ passes the stop line at the downstream intersection

TT: $\quad$ The estimation value of travel time $T T_{i}: \quad i$ th travel time sample $T T_{i}$

$f(T T): \quad$ The probability density function of travel time $T T$

$g\left(T T_{i} \mid \mu_{1}, \sigma_{1}\right)$, The probability density functions of $g\left(T T_{i} \mid \mu_{2}, \sigma_{2}\right)$ : travel time $T T$ of the two distributions, respectively

$p(i, 1), p(i, 2)$ : The conditional distributions of the two distributions when $i$ th travel time is known

$p_{1}, p_{2}: \quad$ The conditional probability of the two distributions, respectively

$T T_{p_{1}=p_{2}}: \quad$ The travel time estimation at the crossing point of the two distributions, namely, the travel time estimation value when $p_{1}=p_{2}$

$m_{k}$ : The number of signal cycles in $k$ th time window

$c_{j}$ : The length of $j$ th signal cycle with $j$ smaller than $m_{k}$

$c: \quad$ The cycle length when the signal timing plan is fixed

$\mathrm{TW}_{k}$ : The value of $k$ th time window

$R_{T T}^{m_{k}}$ : The relative variation ratio of the travel time estimation in $k$ th time window 


$\begin{array}{ll}R_{\text {MBIT }}^{m_{k}:} & \begin{array}{l}\text { The Modified Buffer Time Index in } k \text { th } \\ \text { time window }\end{array} \\ T T_{k 1}: & \text { Travel time estimation of the first signal } \\ & \text { cycle in } k \text { th time window } \\ T T_{m_{k}+1}: \quad \begin{array}{l}\text { Travel time estimations of }\left(m_{k}+1\right) \text { th } \\ \text { signal cycle in } k \text { th time window }\end{array} \\ \text { MBIT }_{k 1}: \quad \begin{array}{l}\text { The Modified Buffer Time Index of the } \\ \text { first signal cycle in } k \text { th time window }\end{array} \\ \text { MBIT }_{m_{k}+1}: \begin{array}{l}\text { The Modified Buffer Time Index of } \\ \left.\text { ( } m_{k}+1\right) \text { th signal cycle in } k \text { th time }\end{array} \\ \text { window } \\ \text { The time difference between the sum of } \\ \text { the two successive time windows and } \\ \text { the minimum response time to traffic } \\ \text { events } \\ \text { The minimum response time to traffic } \\ \text { events. }\end{array}$

Parameters:

$\begin{array}{ll}\mu: & \text { The mean of the travel time distribution } \\ \mu_{1}, \mu_{2}: & \text { The two means of the bimodal } \\ \sigma^{2}: & \text { distribution } \\ \sigma_{1}, \sigma_{2}: & \text { The variance of travel time } \\ & \text { The variances of travel time } \\ \omega_{1}, \omega_{2}: & \text { corresponding to } \mu_{1} \text { and } \mu_{2} \text {, respectively } \\ & \text { The ratio of each subsample to the } \\ & \text { whole sample with the sum of them } \\ \theta(\mu, \sigma, \omega): & \text { equal to } 1\end{array}$

\section{Conflict of Interests}

The authors declare that there is no conflict of interests regarding the publication of this paper.

\section{Acknowledgments}

This work was supported by the National Natural Science Foundation of China (nos. 61304191, 51338008, 51278454, and 51208462), the Fundamental Research Funds for the Central Universities (2014QNA4018), the Projects in the National Science \& Technology Pillar Program (2014BAG03B05), and the Key Science and Technology Innovation Team of Zhejiang Province (2013TD09).

\section{References}

[1] F. Dion and H. Rakha, "Estimating dynamic roadway travel times using automatic vehicle identification data for low sampling rates," Transportation Research B: Methodological, vol. 40, no. 9, pp. 745-766, 2006.

[2] S. W. Dellenback and J. Baumgartner, "Transguide model deployment report," Tech. Rep., Southwest Research Institute, San Antonio, Tex, USA, 2000.

[3] K. C. Mouskos, E. Niver, L. J. Pignataro, S. Lee, N. Antonious, and L. Papadopoulos, "Transmit system evaluation," Final
Report, Institute for Transportation; Institute of Technology, Newark, NJ, USA, 1998.

[4] X. Ma and H. N. Koutsopoulos, "A new online travel time estimation approach using distorted automatic vehicle identification data," in Proceedings of the 11th International IEEE Conference on Intelligent Transportation Systems (ITSC '08), pp. 204-209, Beijing, China, December 2008.

[5] J. Sun and L. Zhang, "Vehicle actuation based short-term traffic flow prediction model for signalized intersections," Journal of Central South University of Technology -English Edition, vol. 19, no. 1, pp. 287-298, 2012.

[6] G.-Y. Jiang, S.-F. Niu, A.-D. Chang, and Y.-L. Cong, "Optimization method of time intervals of data for GPS equipped floating car," Journal of Jilin University (Engineering and Technology Edition), vol. 40, no. 6, pp. 1513-1517, 2010.

[7] W. B. Jackson and J. V. Jucker, "An empirical study of travel time variability and travel choice behavior," Transportation Science, vol. 16, no. 4, pp. 460-475, 1982.

[8] Florida Department of Transportation, The Florida Reliability Method, Florida's Mobility Performance Measures Program, 2000.

[9] C. Chen, A. Skabardonis, and P. Varaiya, “Travel-time reliability as a measure of service," Transportation Research Record, vol. $1855,2003$.

[10] T. Lomax, D. Sharnk, S. Turner et al., Selecting Travel Reliability Measures, U.S. Department of Transportation, FHWA, 2003.

[11] Federal Highway Administration, Travel Time Reliability: Making It There on Time, FHWA, 2006.

[12] J. W. C. Van Lint and H. J. Van Zuylen, "Monitoring and predicting travel time reliability: using width and skew of dayto-day travel time distribution," Transportation Research Record, vol. 1917, pp. 54-62, 2005.

[13] B. Emam and H. M. Al-Deek, "Using real-life dual-loop detector data to develop new methodology for estimating freeway travel time reliability," Transportation Research Record, vol. 1959, pp. 140-150, 2006.

[14] S. Susilawati, M. A. P. Taylor, and S. Somenahalli, "Travel time reliability and the bimodal travel time distribution for an arterial road," Road and Transport Research, vol. 19, no. 4, pp. 37-50, 2010.

[15] K. Jintanakul, L. Chu, and R. Jayakrishnan, "Bayesian mixture model for estimating freeway travel time distributions from small probe samples from multiple days," Transportation Research Record, vol. 2136, pp. 37-44, 2009.

[16] Y. Ji and H. M. Zhang, "Travel time distributions on urban streets: their estimation with a hierarchical 3 Bayesian mixture model and application to traffic analysis using high-resolution bus 4 probe data 5," in Proceedings of the 92nd Annual Meeting of the Transportation Research Board, TRB, Washington, DC, USA, January 2013.

[17] F. Yang, X. Yang, and M. Yun, "Urban road interrupted flow travel time bimodal distribution," Journal of Tongji University, vol. 42, no. 2, pp. 259-265, 2014.

[18] E. Castillo, A. Calviño, M. Nogal, and H. K. Lo, "On the probabilistic and physical consistency of traffic random variables and models," Computer-Aided Civil and Infrastructure Engineering, vol. 29, no. 7, pp. 496-517, 2014.

[19] X. Zhang, H. Liu, and Y. Pu, "Analysis of route travel time variation of road network suffering traffic incidents," Journal of Southwest Jiaotong University, vol. 48, no. 5, pp. 928-933, 2013. 


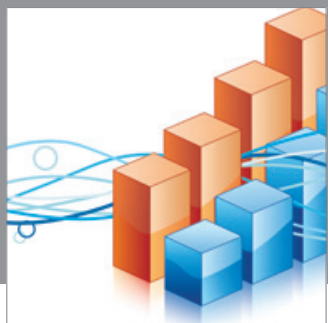

Advances in

Operations Research

mansans

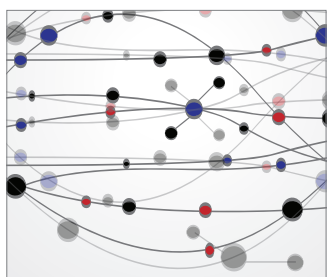

The Scientific World Journal
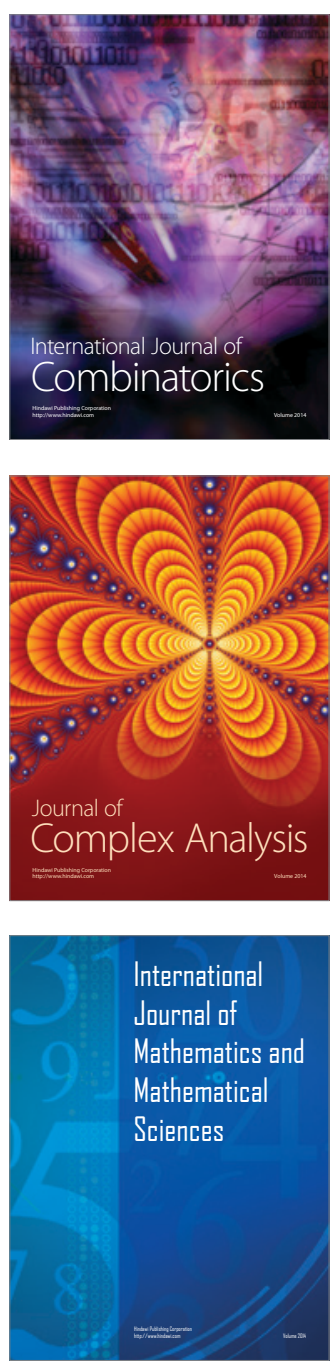
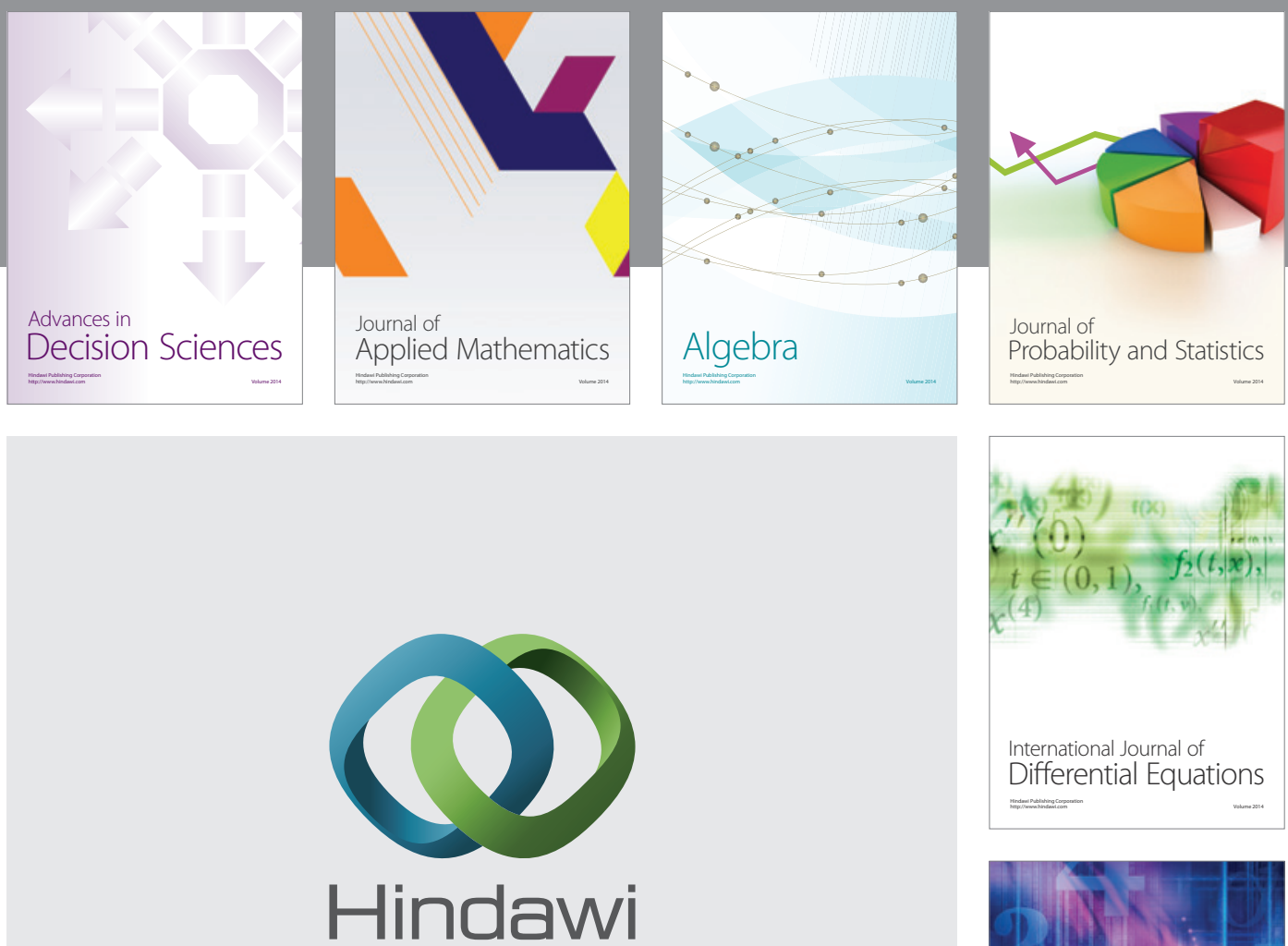

Submit your manuscripts at http://www.hindawi.com
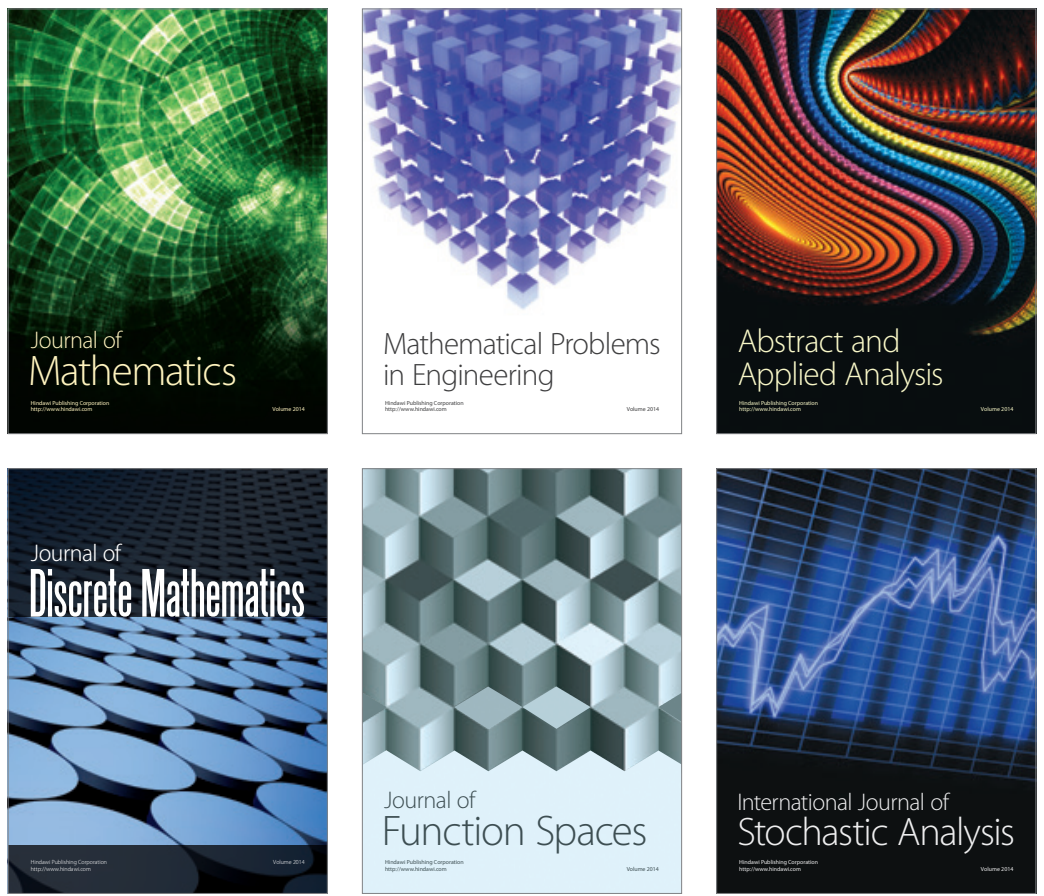

Journal of

Function Spaces

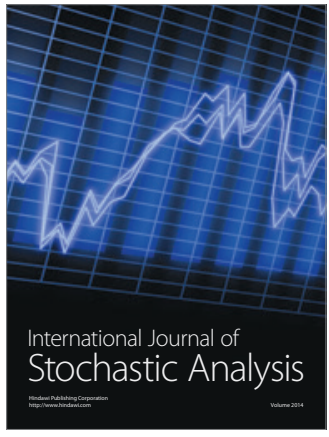

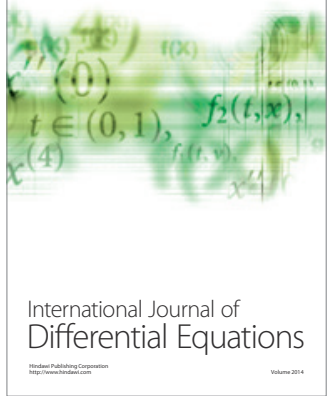
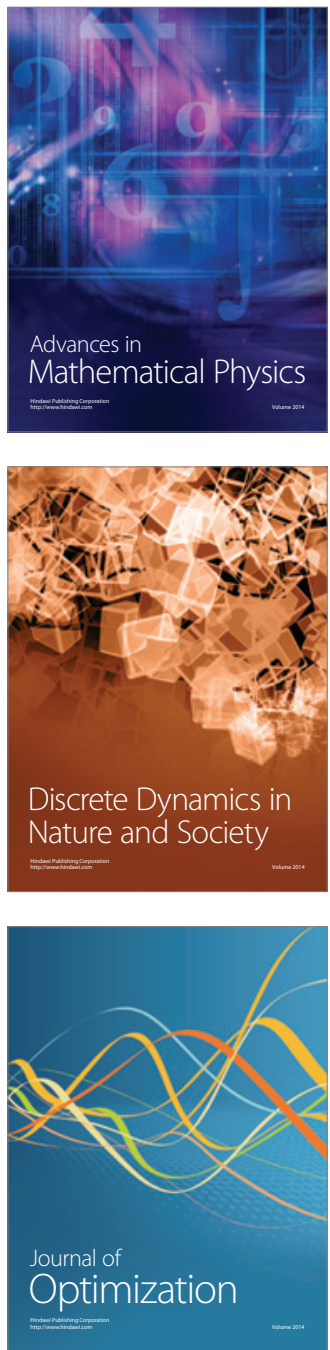